\title{
Urine Specimen Collection For Diagnosing a Urinary Tract Infection-Patient Experience and Preference For Urine Specimen Collection: A Qualitative Study
}

Linda Collins ( $\sim$ I.collins@sgul.kingston.ac.uk)

Kingston University

Rajvinder Khasriya

University College London

James Malone-Lee

University College London

\section{Research Article}

Keywords: MSU, CSU, UTI, Peezy MSU ${ }^{\mathrm{T}}$, Specimen, Collection, Experiences, Preferences

Posted Date: February 13th, 2021

DOl: https://doi.org/10.21203/rs.3.rs-159773/v1

License: (c) (i) This work is licensed under a Creative Commons Attribution 4.0 International License.

Read Full License 


\section{Abstract}

\section{Background}

A urinary tract infection (UTI) is very common and patients frequently present to their General Practitioner and emergency department with symptoms. Diagnosis of the infection is made by examining fresh urine with microscopy, to detect white cells and epithelial cells shed from the bladder or by submitting the urine for microbial culture. Patients are often requested to provide a urine specimen, but very little is known about the patient experiences of carrying out the task or their preference of specimen collection method. The aim of this study was to explore patient experiences and preferences between four different urine specimen collection methods for diagnosing the presence of a UTI.

\section{Materials}

Semi-structured interviews were conducted and explored patient experiences of four different urine specimen collection methods (midstream urine specimen (MSU), catheter specimen (CSU), Peezy MSU ${ }^{\text {TM }}$ and Natural void). The interviews consisted of four open-ended questions and all data were collated and organised using NVivo to establish themes that emerged from the data.

\section{Results}

The natural void without technique was repeatedly referred to and was regarded as being the most straightforward of all of the sampling methods. Pain symptoms were described only in the catheter method and not in the MSU, Peezy MSU ${ }^{\mathrm{Tm}}$ or natural void method. The catheter was regarded as the urine collection method that produced the best specimen for diagnostic testing by the patients'.

\section{Conclusion}

A majority of the participants expressed the desire for straightforward urine sampling methods and preferred the natural void as a standard method in clinical practice.

\section{Background}

A urinary tract infection (UTI) is thought to be caused by microorganisms in the lower urinary tract. $20 \%$ of women will experience a symptomatic UTI in comparison to $10 \%$ of men [1], and prudent antibiotic treatment is the conventional management [2]. A UTI diagnosis accounts for 7 to 8 million clinic visits per year [3]. UTIs can be an incapacitating condition exhibiting lower urinary tract symptoms (LUTS) such as dysuria, urinary frequency, urinary hesitancy and urgency [4].

Clinical guidelines stipulate that patients should provide a urine specimen for microbial culture [1] however, scrutiny of the literature reveals that patient experiences on providing a urine specimen for UTI testing is yet to be explored. When patients attend outpatient consultations they are requested to provide a urine specimen which could be the midstream clean catch technique (MSU) [5], the catheter specimen 
of urine (CSU) [6] or by using a urine collection device commonly known as the Peezy MSU ${ }^{\text {TM }}$ [7]. The willingness to participate in this practice has not been evaluated. The exploration of patient experiences on urine specimen collection has been focused on sexual health screening practices, and data has shown patients do prefer urine specimen testing for diagnosing a sexually transmitted infection (STI) in comparison to the vaginal swabs [8], but patient experiences on urine specimen collection for UTI diagnosis is rare. According to Sheppard [9], patient views and experiences should take precedence in clinical policies and guidelines as it has strengthened patient autonomy when it comes to healthcare provision.

It is understood that patients' choices and preferences promote change in standard practice [10] however, exploring the positive experiences and challenges patients face when asked to provide a specimen of urine is fundamental for future clinical practice. The aim of this study was to explore patient experiences and preferences between four different urine specimen collection methods for diagnosing the presence of a UTI.

\section{Methods}

The qualitative interview study which began in January 2015 for 12 weeks was granted ethical approval by the national research ethics service committee (NRES) London-Harrow. Patients with lower urinary tract symptoms (LUTS) attending a medical urology centre in North London made up the participant population. Convenience sampling was used to select the participants [11]. Participants were drawn from female patients diagnosed with chronic UTI, painful bladder syndrome (PBS), overactive bladder (OAB), general LUTS and who had experience of providing a urine specimen by four different methods which were intermittent catheterisation (CSU), midstream urine (MSU), Peezy MSU ${ }^{\mathrm{TM}}$ as well as natural void with no technique. These specimen collection methods had been conducted when attending the centre for consultation and follow-up review. Patients were provided with instructions for the MSU, Peezy MSU ${ }^{\mathrm{TM}}$ and natural void specimen collection methods (Supplementary file 1) during their attendance at the centre, the CSU method was thoroughly explained. It was a requirement that their experience of providing a urine specimen was within the last three months leading up to the study for vivid recollection of events and this was confirmed from previous urine specimen requests. Patients were given an information sheet about the study and were offered the opportunity to ask questions and address any concerns. The patients who were willing to be interviewed, signed a consent form and were allocated a four-digit non-identifiable participant number. The interviews questions were developed and based upon existing practices of urine specimen collection methods at the medical urology centre. Semi-structured interviews consisted of four open-end questions all of which were supported with rationale (Table 1). The four questions were tested with colleagues at the medical urology centre prior to data collection to check the wording of the questions, the understanding of what was being asked and the appropriateness of interview skills. All interviews were condcuted face to face and data were audio recorded and later transcribed. The data were inputted into NVivo, which is a software that organises unstructured data [12]. All data were anaylsed using the Braun and Clarke's [13] thematic anaylsis method which reveals the emerging themes 
that derive from the data. The interviews were conducted by a doctoral clinical research nurse, who was experienced in conducting interviews for qualitative research.

\section{Results}

We interviewed 30 participants, each lasting approximately 30 minutes. Four main themes were identified when examining patient experiences of providing a urine specimen for diagnosing a UTI (Table 2).

\section{Theme 1: Straight forward urine collection}

For interview question 1, each participant spoke about being able to provide a urine sample that was easy and straightforward. The naturally voided method was repeatedly referred to and was regarded as being the most straightforward of all of the sampling methods. Many of the participants talked about being able to provide a urine specimen that was not labour intensive or painful. Participants shared their views on natural voiding by referring to the collection method as "easy" (Participant 3867). However, other participants were more explicit when describing the method.

"It's not a problem you just open your legs and let it all out"(Participant 3458).

"I prefer urinating into the pot. It's straightforward" (Participant 2365).

Participants often mentioned being able to provide a urine sample that was quick and easy, especially as LUTS were the primary reason for them attending the medical urology centre. The MSU and the Peezy method were not considered straightforward compared to the natural void. There were mixed views towards the MSU as some participants found the MSU method challenging and expressed concerns.

"You wonder whether you have done it right" (Participant 2906).

"The midstream is awkward because you don't know when you catching the midstream"(Participant 2287).

"The midstream I don't like because if you lose the urine whilst getting the midstream you lose it all over your hands" (Participant 3121).

Straightforward urine collection was considered the best way for obtaining a urine specimen. It was clearly established that participants were more enthusiastic about providing a urine specimen that was easy and straightforward to perform. Although they were familiar with the MSU, there remained uncertainties with the collection technique.

The Peezy MSU ${ }^{\mathrm{TM}}$ was regarded as complex and was not the easiest method for obtaining a urine sample and participants frequency expressed their views on the complexities of the device.

"I wasn't keen on the peezy as I needed an extra pair of hands, and I didn't trust the instruction provided in the pack" (Participant 3600). 
"I didn't like the peezy because I couldn't work out how it worked and I was worried it was going to disconnect and I would end up peeing all over the floor" (Participant 4014).

"The MSU peezy; It was confusing; I needed a demonstration of how to use the device. I wasn't sure whether I was to stand up or sit down when using the device" (Participant 4265).

\section{Theme 2: Painful urine specimen collection}

As a majority of the participants attending the centre had painful bladder symptoms, the experience of having the catheter inserted into the bladder brought back the memory of that pain. For interview question 2, participants commonly described their experiences of being catheterised as painful and uncomfortable. The pain symptoms were described only in the catheter method and not in the MSU, Peezy MSU ${ }^{\mathrm{TM}}$ or natural void methods as these methods were all non-invasive. Participants described their dislike towards the catheter method.

"I don't particularly like the catheter because it's scary even though it's not and feels as though you might get an infection" (Participant 3499).

"It's slightly uncomfortable. When it's taken out it has a strange sensation"(Participant 2584).

The majority of the participants experienced a great deal of pain with the catheter collection method. In addition it infringed on their privacy due to the way in which the specimen was collected, and participants highlighted this point.

"The catheter was a bit invasive and it hurt" (Participant 2977).

As the catheter technique was believed to produce the optimal specimen, the pain associated with the method was endured for the purpose of obtaining an uncontaminated specimen.

\section{Theme 3: The optimal specimen collection}

The catheter was regarded as the urine collection method that produced the best specimen for diagnostic testing. When asked, what method of urine collection do you think provided the most clean sample and why? The participants responded positively.

"The catheter of course. Because it's not touching anything to pick up any germs"(Participant 3458).

"Probably the catheter because your introducing it straight into the urethra and there are no chances of germs entering"(Participant 3963).

A majority of the participants were adamant that the catheter was a better method and could not compare to the non-invasive methods. Although there were no overt claims that the catheter specimen was less likely to contain contaminants, there were subtle remarks suggesting that the catheter is a superior method. 
"The catheter went straight in and eliminated areas where it could get contaminated" (Participant 3600).

"The catheter provided the cleanest sample as it is all internal and it's the cleanest way of collecting the sample"(Participant 4014).

"I would have thought that the catheter would provide the opportunity to be uncontaminated, and provide the cleanest sample" (Participant 3456).

As more interviews were conducted it emerged that participants thought that the method of how the catheter was inserted into the bladder would bypass the chances of specimen contamination and because of this, strongly believed that the catheter sampling method was the optimal specimen for identifying a UTI.

Some of the participants did not consider the other three methods could compete with the catheter and shared their experiences. There was a strong census that the catheter specimen was a superior specimen collection method and this was evident through the comments of each participant.

"Surely the other methods were not as good at getting a cleaner sample like the catheter?" (Participant 4300).

"There is a likelihood of contamination from the other methods"(Participant 2131).

"The other methods would most probably pick up germs, which the catheter wouldn't" (Participant 3458).

\section{Theme 4: Simple urine collection methods as standard practice}

Participants' agreed that they would like a urine collection method that would be easy and straightforward to perform. A majority of the participants considered that the natural void should be the standard method in clinical practice for patients without a complex bladder, unlike them who require specialist treatment interventions.

\section{Discussion}

The participants were known to have a chronic UTI and LUTS. The interviews revealed how important it was for them to have an uncontaminated urine specimen that would show the true pathology of their disease. The participants thought that because the catheter was inserted directly into the bladder it provided a true identification of the causative pathogens unlike the non-invasive methods. The catheter specimen was considered the optimal method for obtaining a urine specimen free from external contamination and was the best method for them as they had a chronic urine infection accompanied with complex bladder symptoms. The reassurance of knowing that a urine collection method would provide an accurate identification of a UTI was considered an important factor, and in this case the catheter was the best method. The participants did not believe that the other non-invasive methods were of the same calibre and were not trusted to scrutinise the microbes that were invading the urinary tract. 
Recent data has revealed that this was not the case [14] and that the catheter specimen actually bypassed the cells and sediments that were at the base of the bladder, which were crucial for diagnosing the offending microbes [14].

Despite the pain, there was a strong belief that the catheter method was best for identifying the UTI. Although pain and discomfort were closely associated with having a urine specimen collected by the catheter, the pain was the ultimate price to pay for an uncontaminated specimen. The participants were known to have a urinary tract infection, and some had urethral inflammation, as a secondary symptom, at the time the catheter was being inserted into the bladder. Urethritis is the inflammation of the urethra and is commonly diagnosed in patients with a urinary tract and sexually transmitted infection [15]. Having the catheter inserted with the presence of urethritis triggered further pain symptoms. Although the participants may not have had a comprehensive understanding of the pathophysiological aspects of a urinary tract infection, they were more aware of the pain and discomfort associated the catheter method compared to the other three non-invasive methods. The participants focused on having a urine specimen that was uncontaminated and that would identify the true pathology of a urinary tract infection. With the intention of obtaining an optimal specimen, the participants would rather endure the pain associated with the catheter method, than to have a contaminated specimen from the MSU, Peezy MSU ${ }^{\mathrm{TM}}$ and natural void methods. The natural void was the most desired urine collection method as it was straightforward but was not considered the appropriate method for those who had LUTS, a UTI and required an accurate pathology of their infection.

The participants expressed that the natural void was straightforward and very easy to perform. They were not required to wipe the perineal area prior to voiding which many considered was a natural way to urinate. The natural void method was considered effortless and a well-suited urine collection method for anybody providing a urine specimen. The participants felt the need to express their views and experiences of urine collection methods that were not straightforward. They were vocal when describing the problems faced when collecting a MSU and when using the Peezy MSU ${ }^{T M}$ device. The difficulty with the midstream method was that participants often reported that they were unsure which part of the urinary stream was the middle, and accurate judgement of this remained a constant concern. That was not the only unease related to the midstream method; fear of soiling hands during the collection process and having to wipe the perineal area brought on anxieties of whether the wiping method was correct or not. There were apprehensions towards the Peezy MSU ${ }^{\mathrm{TM}}$ device as participants often reported their lack of understanding on how to use it, as well as discussing their fears of the device being disconnected during the voiding process. These were anxieties and concerns raised when it came to providing a specimen in ways other than the natural void method.

\section{Conclusion}

With an increased number of patients asked to provide a urine specimen in busy outpatient settings for urinary diagnostics, the natural void method undoubtedly would seem to ease the pressures on clinical staff who explain urine collection techniques to patients. The importance of patient experiences is that it 
influences changes in clinical practice [10]. The interview data revealed that participants collectively believed that the natural void was a well-suited method for urine collection in standard general practice. The ability to provide a urine specimen that was simple and without a technique was favoured. The participants thought the natural void method had these attributes and believed that it should be implemented as standard general practice for specimen collection. Being able to provide a specimen that was easy to perform was the specimen method of choice. However, the natural void method was regarded a better option for patients who did not have a chronic urinary tract infection or a complex bladder.

\section{Abbreviations}

CSU: Catheter specimen

LUTS: Lower urinary tract symptoms

MSU: Midstream urine specimen

NRES: National research ethics service committee

OAB: Overactive bladder

PBS: Painful bladder syndrome

Peezy MSU ${ }^{\text {Tw}}$ : midstream urine specimen

STI: Sexually transmitted infection

UTI: Urinary tract infection

\section{Declarations}

\section{Ethics approval and consent to participate}

Ethical approval was granted by the national research ethics service committee (NRES) London-Harrow in accordance with the Helsinki ethical principles for research involving human subjects. The primary purpose of this study was to explore patient experiences and preferences between four different urine specimen collection methods for diagnosing the presence of a UTI with lower urinary tract symptoms (LUTS). The study was carried out in accordance with the study protocol presented and accepted by the NRES.

\section{Consent for publication}

All participants provided informed consent to participant in the study and for publication of study findings. 


\section{Availability of data and materials}

The datasets used and/or analysed during the current study available from the corresponding author on reasonable request. Data has been made available to the national research ethics service committee, medical urology centre and patients at request.

\section{Competing interests}

There were no competing interests.

\section{Funding}

There were no funding or incentives for conducting the study.

\section{Authors contributions}

Collins: Protocol/project development, data collection, management of data analysis, manuscript writing/editing.

Khasriya: writing/editing.

Malone-Lee: Protocol/project development, management of data analysis, writing/editing.

\section{Acknowledgements}

We would like to acknowledge all the patients for their participation in the study, the nurse, doctors and laboratory staff at the medical urology centre who contributed their time and supported the study in various ways.

\section{References}

1. NICE, Urinary tract infections in adults, N. England, Editor. 2015, National Institute for Health and Care Excellence.

2. Asma, B., et al., Standardised high dose versus low dose cranberry Proanthocyanidin extracts for the prevention of recurrent urinary tract infection in healthy women [PACCANN]: a double blind randomised controlled trial protocol. BMC Urol, 2018. 18(1): p. 29.

3. Robinson, D., I. Giarenis, and L. Cardozo, The management of urinary tract infections in octogenarian women. Maturitas, 2015. 81(3): p. 343-7.

4. Bono, M.J. and W.C. Reygaert, Urinary Tract Infection, in StatPearls. 2018, StatPearls Publishing StatPearls Publishing LLC.: Treasure Island (FL).

5. Dougherty, L., S. Lister, and A. West-Oram, The Royal Marsden Manual of Clinical Nursing Procedures (Royal Marsden Manual Series). 9th Edition ed. 2015, West Sussex: Wiley Blackwell. 
6. Iggulden, H., C. MacDonald, and K. Staniland, Clinical skills: the essence of caring. 2009, Berkshire: Open University Press.

7. Collier, S., et al., A prospective study comparing contamination rates between a novel mid-stream urine collection device (Peezy) and a standard method in renal patients. Journal of Clinical Pathology., 2013. 67(2): p. 139-142.

8. Bilardi, J.E., et al., Young pregnant women's views on the acceptability of screening for chlamydia as part of routine antenatal care. Bmc Public Health, 2010. 10.

9. Sheppard, M.K., Fallacy or Functionality: Law and Policy of Patient Treatment Choice in the NHS. Health Care Analysis, 2014. 10.1007/s10728-014-0275-6.

10. Tebb, K.P., et al., Home STI testing: The adolescent female's opinion. Journal of Adolescent Health, 2004. 35(6): p. 462-467.

11. Moule, P.P.a., H.D.a. Aveyard, and M.a. Goodman, Nursing Research: An Introduction. Third Edition ed. 2017.

12. QSR, I. What is Nvivo? 2016 [cited 20168 August 2016]; Available from: http://www.qsrinternational.com/what-is-nvivo.

13. Braun, V. and V. Clarke, What can "thematic analysis" offer health and wellbeing researchers?, in Int J Qual Stud Health Well-being. 2014: Sweden. p. 26152.

14. Collins, L., et al., A revalidation and critique of assumptions about urinary sample collection methods, specimen quality and contamination. Int Urogynecol J, 2020. 31(6): p. 1255-1262.

15. Moi, H., K. Blee, and P.J. Horner, Management of non-gonococcal urethritis. BMC Infect Dis, 2015. 15(1): p. 294.

\section{Tables}

Table 1: This table lists the semi-structured interview questions all 30 participants were asked supported with rationale for each of the questions. 


\begin{tabular}{|lll|}
\hline $\begin{array}{l}\text { Question } \\
\text { number }\end{array}$ & Question asked & Rationale for question \\
$\begin{array}{l}\text { Interview } \\
\text { question }\end{array}$ & $\begin{array}{l}\text { Which method of } \\
\text { urine collection did } \\
\text { you prefer and why? }\end{array}$ & $\begin{array}{l}\text { This question was asked because participants remember } \\
\text { sampling methods they prefer rather than sampling methods } \\
\text { that are least preferred. }\end{array}$ \\
\hline $\begin{array}{l}\text { Interview } \\
\text { question }\end{array}$ & $\begin{array}{l}\text { Which method of } \\
\text { urine collection did } \\
\text { you not like and why? }\end{array}$ & $\begin{array}{l}\text { This question was asked to establish which method of urine } \\
\text { specimen collection was least preferred and what their reasons } \\
\text { were. }\end{array}$ \\
\hline $\begin{array}{l}\text { Interview } \\
\text { question }\end{array}$ & $\begin{array}{l}\text { What method of urine } \\
\text { collection do you } \\
\text { think provided the } \\
\text { cleanest sample and } \\
\text { why? }\end{array}$ & $\begin{array}{l}\text { The patients attending the medical urology centre are aware } \\
\text { that a clean uncontaminated urine specimen is required when } \\
\text { diagnosing a UTI. This question aimed to explore the } \\
\text { participants' perception of optimal specimen collection. }\end{array}$ \\
\hline $\begin{array}{l}\text { Interview } \\
\text { question }\end{array}$ & $\begin{array}{l}\text { Which method of } \\
\text { urine collection do } \\
\text { you think should be } \\
\text { used as a standard } \\
\text { method and why? }\end{array}$ & $\begin{array}{l}\text { Patient preferences and choice should influence the way } \\
\text { screening and diagnostic tests are to be implemented. This } \\
\text { question was included to explore how patient experiences might } \\
\text { influence change in the future of specimen collection and } \\
\text { diagnostic testing. }\end{array}$ \\
\hline
\end{tabular}

Table 2: This table highlights the four main themes identified when patients talked about their experiences of providing a urine specimen.

\section{Data Themes}

Theme 1: Straightforward urine collection

Theme 2: Painful urine specimen collection

Theme 3: The optimal specimen collection

Theme 4: Simple urine collection methods as standard practice

\section{Supplementary Files}

This is a list of supplementary files associated with this preprint. Click to download.

- AdditionalFile1.docx

- Supplementaryfile1.docx 